\title{
Photocatalytic oxidation of stilbene by self-assembled stacks of manganese porphyrins
}

\author{
Miriam de Torres,* Richard van Hameren, Johannes A.A.W. Elemans, Alan E. Rowan and Roeland J.M. \\ Nolte
}

\section{DOI: $10.1039 / b 000000 x$}

Self-assembled stacks of trimeric manganese porphyrins exhibit high activity in the photocatalytic epoxidation of stilbene using $\mathrm{O}_{2}$ as oxidant. The supramolecular structure of 10 the catalyst leads to increased stability of the system and enhances the stereoselectivity of the reaction.

The use of light as the activation energy for photochemical reactions is being extensively investigated due to the availability of this inexpensive and clean natural resource. ${ }^{1}$ An ideal 15 component of such a photodriven chemical process is a porphyrin. They are attractive compounds for applications in photochemical reactions, in particular oxidation reactions because they absorb strongly in the visible spectrum and their properties can be easily tuned by attaching different substituents to the 20 macrocycle and/or inserting different metal centers. ${ }^{2}$ It has been reported that such metal catalysts can readily epoxidise alkenes with molecular oxygen as oxidant and an aldehyde as coreductant. ${ }^{3}$ Mechanistic studies have shown that the epoxidation of alkenes by $\mathrm{O}_{2}$ in the presence of Mn-porphyrins and 25 isobutyraldehyde occurs via a free radical mechanism through a high-valent metal oxo intermediate, which reacts with the alkene. ${ }^{4}$ Here, we report on a new supramolecular catalyst (MnTAP, see Fig.1), based on a 1,3,5-benzene tricarboxamide (BTA) core, substituted with three Mn porphyrins, for the photocatalytic 30 epoxidation of cis-stilbene with $\mathrm{O}_{2}$. BTA derivatives have been shown to self-assemble in organic solvents into helical supramolecular stacks stabilised by a three fold hydrogen bonding network and л-л- stacking interactions. ${ }^{5}$ Structurally similar TAP has been shown to form well-defined columnar ${ }_{35}$ stacks in both solution and at a surface, ${ }^{6}$ Fig. 1. Molecular modelling and scanning tunnelling microscopy (STM) studies of these stacks point to the presence of small spaces between adjacent porphyrins in these stacks, ideal for substrate selective catalysis. Assuming that Mn-TAP self-assembles in a similar way

40 to its metal-free derivative, we decided to use theses spaces as cavities in which an alkene can bind and be catalytically oxidised by the proximal manganese catalyst. It has also been previously shown that the introduction of a bidentate ligand such as 1,4diaza $[2,2,2]$ bicyclooctane (DABCO) to the TAP system can 45 influence the stability and number of cavities within a stack. This ligand can bind in a sandwich-like fashion between two neighbouring Mn-porphyrin moieties electronically

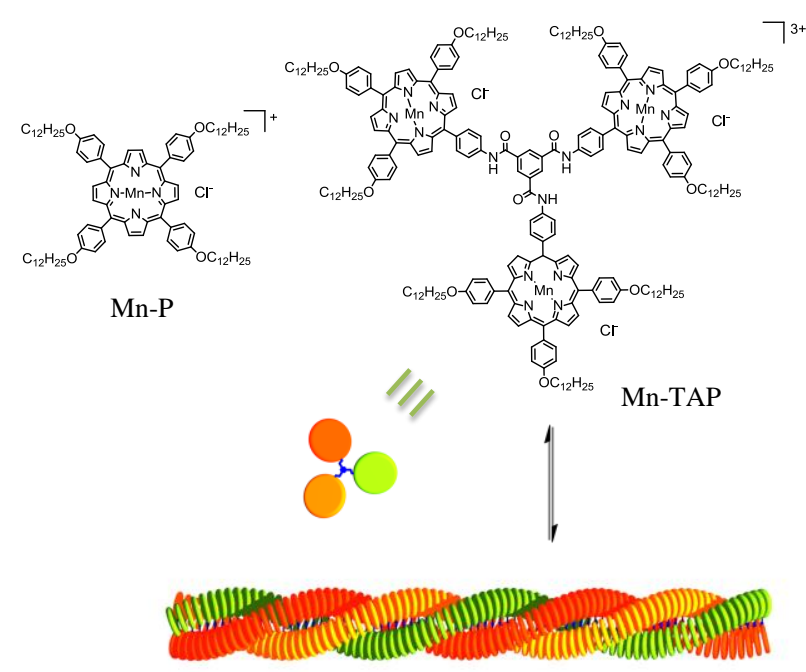

Fig. 1 Chemical structures of the catalysts Mn-P and Mn-TAP. Schematic representation of the proposed columnar stack of Mn-TAP. Chloride atoms are present in the manganese catalysts as counterions.

Table 1 Light effect in the photocatalytic epoxidation of cis-stilbene by Mn-TAP.

\begin{tabular}{ccccc}
\hline & Temperature $\left({ }^{\circ} \mathrm{C}\right)$ & Light & Time (min) & Conversion $(\%)$ \\
\hline 1 & 20 & Xe-lamp & 10 & 69 \\
2 & 20 & Xe-lamp & 30 & 100 \\
3 & 20 & - & 120 & 0 \\
4 & 20 & Sunlight & 10 & 19 \\
5 & 20 & Sunlight & 60 & 50 \\
6 & 40 & Sunlight & 10 & 46 \\
7 & 40 & Sunlight & 60 & 100
\end{tabular}

${ }^{a} 0.125 \mathrm{mmol}$ cis-stilbene, $0.1 \% \mathrm{~mol}$. Mn-TAP, $\mathrm{O}_{2}, 1 \mathrm{ml} \mathrm{CH}_{2} \mathrm{Cl}_{2}, 5$ equiv. 55 of isobutyraldehyde, 2 equiv. DABCO.

activating the catalyst.

Experiments were carried out with cis-stilbene as a model substrate and the effects of the reaction conditions, including the 60 effect of light, on its epoxidation with molecular oxygen as oxygen donor and isobutyraldehyde as oxygen carrier in the presence of Mn-TAP were investigated. For comparison, reactions were also performed with the tetrasubstituted monomeric Mn-porphyrin, Mn-P (Fig. 1). The amount of ${ }_{65} \mathrm{DABCO}$ was fixed to 2 equivalents with respect to $\mathrm{Mn}$ centres 
and the reactions were carried first using a Xenon lamp (150 W, spectral distribution $240-2000 \mathrm{~nm}$ ) as intense light source and at room temperature. Under these standard conditions, cis-stilbene was totally converted with $0.1 \%$ mol. of Mn-TAP.

5 The turnover frequency of the catalyst (TOF, measured when the substrate was totally consumed) was $32 \mathrm{~min}^{-1}$. ${ }^{1} \mathrm{H}$ NMR analysis of the crude reaction mixtures confirmed that the oxidation reaction was completely chemoselective towards epoxides, without the occurrence of ring-opening side reactions 10 (Fig. 1S).

Control experiments to analyse the influence of light, catalyst, and the presence of isobutyraldehyde were performed (Table 1). Light was found to be crucial for the epoxidation to occur: no conversion of alkene was observed in the dark at room 15 temperature. Slower reactions were observed under ambient sunlight conditions, even when the reaction mixture was heated to $40{ }^{\circ} \mathrm{C}$ (Table 1). It is clear that light enhances the rate of the catalytic reaction. In the absence of Mn-TAP, no significant substrate conversion occurred and with only $2 \%$ epoxide 20 produced, indicating that the presence of a porphyrin is important for the epoxidation reaction to occur efficiently. The small amount of product formed can be attributed to a reaction between the alkene substrate and radicals generated from the autooxidation of the aldehyde. ${ }^{4}$ It was also observed that no epoxide 25 was obtained in their absence of either isobutyraldehyde or molecular oxygen. Experiments carried out in air and ambient sunlight, resulted in slower reaction rates, yielding only $15 \%$ of epoxide after $10 \mathrm{~min}$ and $41 \%$ after $60 \mathrm{~min}$.

The results indicate that the catalyst is activated by light, 30 probably by a photoexcited state on the porphyrin that allows the activation of the oxygen at room temperature. The mechanism of alkene epoxidation by $\mathrm{O}_{2}$ in the presence of metal porphyrins and aldehyde as an oxygen acceptor has been previously investigated. ${ }^{4}$ It was postulated that the reaction proceeds via a 35 free radical mechanism. In order to prove the presence of radicals in our system using Mn-TAP as a catalyst, a radical inhibitor (2,2,6,6-tetramethyl-1-piperidinyloxy, TEMPO, $0.625 \mathrm{mmol}$ ) was added to the epoxidation reaction mixture under the standard conditions. In agreement with early studies, the epoxidation of 40 cis-stilbene was fully quenched. Although it is known that porphyrins and phthalocyanines, especially the zinc derivatives, can act as photosensitizers and trigger the formation of ${ }^{1} \mathrm{O}_{2},{ }^{7}$ this was not observed when manganese-corroles were used as catalysts in the photocatalytic oxidation of $\mathrm{PPh}_{3}{ }^{8}$ The presence of 45 oxygen radicals can be probed using 9,10-dimethylalthracene (DMA). ${ }^{9}$ The reduction in the intensity of bands between 300 and $400 \mathrm{~nm}$ in the UV-visible absorption spectra of DMA, should be observed if ${ }^{1} \mathrm{O}_{2}$ is present. Following this approach, Mn-TAP $\left(10^{-}\right.$ ${ }^{5} \mathrm{M}$ ) and (DMA $10^{-3} \mathrm{M}$ ) were dissolved in dichloromethane in an 50 oxygen atmosphere, the mixture was irradiated with a Xenon lamp for $1 \mathrm{~min}$. Substantial changes in the UV-vis absorption spectra of the mixture before and after the exposure to light and oxygen were observed (Fig. 2S). After the exposure the spectra showed the loss of all peaks belonging to DMA, suggesting that ${ }_{55}{ }^{1} \mathrm{O}_{2}$ is involved in the oxidation process.

Dynamic Light Scattering (DLS) measurements were performed to investigate whether Mn-TAP displayed the same self-assembly behaviour as the previously reported metal-free porphyrins trimers. ${ }^{6}$ According to DLS, a homogeneous solution ${ }_{60}$ of Mn-TAP in cyclohexane with various amounts of DABCO present (0-10 equivalents), contained aggregates with an average size of $350 \mathrm{~nm}$ (Table 1S). In all cases the polydispersity index was high indicating a mixture of different stack lengths. The same Mn-TAP-DABCO mixtures in dichloromethane contained 65 much shorter aggregates, which were not detectable by DLS. These studies demonstrate that the polarity of the solvent has a profound influence on the length of the stacks, because a decreasing in solvent polarity gives rise to stronger intermolecular hydrogen bonding and desolvation of the aromatic 70 porphyrin planes. The presence of a Mn centre in the porphyrins is expected to result in a decrease in the length of the stack compared to metal free porphyrin trimers due to the presence of the axially coordinating chloride ligand, which hinders the selfassembly process.

75 Having shown that the Mn-TAP indeed forms large assemblies in solution, the effect of the supramolecular structure on the epoxidation reaction was analysed in terms catalyst stability and trans/cis product stereoselectivity. To test the stability of the catalyst, Mn-TAP was exposed to molecular oxygen and light 80 under standard conditions in dichloromethane and cyclohexane. Under the above conditions, Mn-TAP was found to be stable for 2 hours in an oxygen atmosphere in the dark at room temperature, but it was found to slowly decompose under intense light, as shown by the changes in the absorption spectrum (Fig. 3S, 4S). ${ }_{85}$ The Soret band $(481 \mathrm{~nm})$ and the broad bands between (320 and $430 \mathrm{~nm}$ ) gradually decreased in intensity. This decrease was oxygen independent and was not recovered after keeping the reaction mixture for $2 \mathrm{~h}$ in the dark again, followed by exposure to high intensity.

90 In the presence of DABCO, a bifunctional axial ligand, UV-vis experiments revealed that in both solvents the stability of the catalyst was increased at higher concentrations (Fig. 5S). The Mn-TAP was more stable in cyclohexane than in dichloromethane. For comparison, the stability of molecularly 95 dissolved Mn-P was studied under the same conditions. In comparison with Mn-TAP, the decomposition of Mn-P was found to be concentration dependent, but significantly higher in all cases (Fig. 6S).

In order to study the effect of the supramolecular structure of 100 the catalysts on their stereoselectivity, the photocatalytic epoxidation of cis-stilbene was carried out with Mn-TAP and $\mathrm{Mn}-\mathrm{P}$ in cyclohexane and dichloromethane, in the presence of $\mathrm{DABCO}$ as an axial ligand.

As shown in Table 2, in both solvents trans-stilbene oxide was 105 the main product when Mn-TAP was used as a catalyst. The reaction rate and the final epoxide yield were higher with Mn-P as catalyst compared to Mn-TAP in dichloromethane. The ratio trans/cis epoxide however is in both cases higher when Mn-TAP is used as a catalyst. Intriguingly, the larger aggregates of Mn110 TAP, which are present in cyclohexane, lead to a significantly higher trans/cis stereoselectivity. In addition, the trans-epoxide is the dominant product even was at low conversion values (Table 2 ). We propose that the stereoselectivity of the trans-stilbene oxide originates from the confined reaction spaces present in the 115 stacks of Mn-TAP. The olefin interacts with the Mn centres and is then converted into the less bulky product (Fig. 2). ${ }^{10}$ 
Table 2 Effect of the supramolecular structure in which the catalyst can self-assemble on the stereoselectivity of the photocatalytic epoxidation of cis-stilbene by $\mathrm{O}_{2}$ in the presence of Mn-TAP or Mn-P.

\begin{tabular}{ccccc}
\hline Catalyst & \multirow{2}{*}{ Solvent } & Time (min) & Conversion (\%) & Trans/Cis \\
\hline Mn-TAP & $\mathrm{CH}_{2} \mathrm{Cl}_{2}$ & 10 & 69 & 2.4 \\
Mn-TAP & $\mathrm{CH}_{2} \mathrm{Cl}_{2}$ & 30 & 100 & 2.1 \\
MnP & $\mathrm{CH}_{2} \mathrm{Cl}_{2}$ & 10 & 78 & 1.3 \\
MnP & $\mathrm{CH}_{2} \mathrm{Cl}_{2}$ & 30 & 100 & 1.3 \\
Mn-TAP & Cyclohexane & 10 & 20 & 7.3 \\
Mn-TAP & Cyclohexane & 30 & 27 & 4.9 \\
MnP & Cyclohexane & 30 & 30 & 1.1 \\
MnP & Cyclohexane & 60 & 41 & 1.3
\end{tabular}

${ }^{a} 0.125 \mathrm{mmol}$ cis-stilbene, $0.1 \%$ mol. Catalyst, $\mathrm{O}_{2}, 2$ equiv. DABCO, 1 $5 \mathrm{ml}$ solvent, 5 equiv. isobutyraldehyde, RT, irradiation with a Xenon lamp.

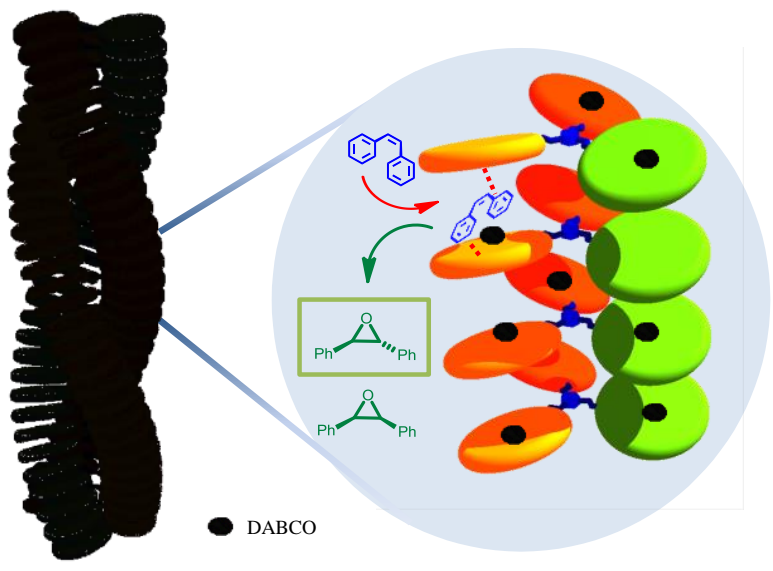

Fig. 2 Caption Proposed schematic model of part of a catalytic stack of Mn-TAP including DABCO as axial ligands and the way cis-stilbene enters the stacks and subsequently reacts in the confined spaces between 10 the porphyrins.

According to this hypothesis, higher ratios of trans/cis stilbene oxide should be generated in solvents that contribute to the formation of larger aggregates which is in line with the results shown in Table 2. If olefin has to penetrate the catalytic stack 15 then the reaction is expected to be slower than in the case of molecularly dissolved porphyrin catalysts, where such a steric hindrance is absent. This effect is also observed (Table 2).

The influence of DABCO on the catalytic performance of MnTAP is shown in Fig. 3. By increasing the amount of DABCO 20 with respect to Mn-TAP the stacks become shorter according to the DLS measurements (Table 1S). In the absence of DABCO, more epoxide is formed in both solvents, but stereoselectivity is almost absent. In cyclohexane, an increase in the amount of DABCO leads to a gradual decrease in the yield of epoxide, but 25 at the same time the selectivity for the formation of trans-stilbene oxide becomes far more pronounced, again in line with the confined cavity hypothesis. In dichloromethane, where the stacks are much shorter or even completely absent, the epoxide yield remains constant up to the addition of 2 equivalents of DABCO 30 and then gradually decreases. At the same time, the trans/cis ratio only slightly increases, which is in stark contrast to the selectivity observed in case of catalysis using Mn-TAP in cyclohexane.

In conclusion, we have developed a new photocatalytic selfassembled system composed of manganese porphyrin trimers,

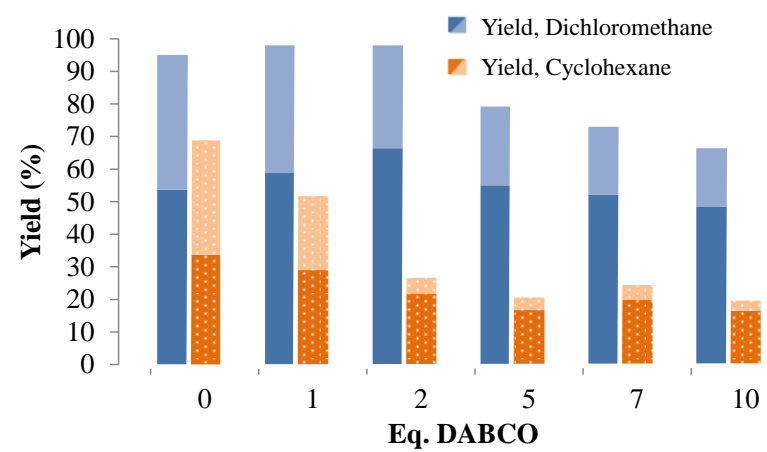

Fig. 3 Effect of the amount of axial ligand DABCO on the yield and stereoselectivity of the photocatalytic epoxidation of cis-stilbene with molecular oxygen catalysed by Mn-TAP. Trans/cis stilbene-oxide ratio is defined through dark (trans)/clear (cis) colour ratio for both solvents.

40 which has been used for the epoxidation of alkenes with molecular oxygen as oxidant. In the supramolecular stacks formed by the catalyst, confined spaces are located between the porphyrins molecules. These small cavities enhance the stereoselectivity of the reaction in the sense that predominantly 45 trans-stilbene oxide is formed, not the energetically favourable cis-stilbene. Current studies are aimed at driving selective epoxidation of alkene mixtures with manganese porphyrin trimers by hindering the access of more bulky substrates, using different axial ligands which can tune the size of the cavities.

\section{Notes and references}

${ }^{a}$ Radboud University Nijmegen, Institute for Molecules and Materials, Heyendaalseweg 135, 6525 AJ, Nijmegen, The Netherlands. Fax: XX XXXX XXXX; Tel: XXXXXXXXXX; E-mail: xxxx@aaa.bbb.ccc

$55 \dagger$ Electronic Supplementary Information (ESI) available: [details of any supplementary information available should be included here]. See DOI: $10.1039 / \mathrm{b} 000000 \mathrm{x} /$

$\$$ It should also be noted that $c i s$-stilbene did not isomerize to transstilbene under the influence of light, consequently any formed trans60 stilbene oxide arose from the catalytic process.

$\ddagger$ Reaction temperature under intense light was $20^{\circ} \mathrm{C}$.

In order to investigate if the activation of light is caused by thermal effects as a result of the irradiation, the epoxidation reactions were carried out at $40{ }^{\circ} \mathrm{C}$ in the absence of light. Only $17 \%$ of cis-stilbene was 65 converted during the first $10 \mathrm{mins}$, and $63 \%$ after $30 \mathrm{~min}$.

1. a) M. Schulz, M. Karnahl, M. Schwalbe, and J. G. Vos, Coord. Chem. Rev., 2012, 256, 1682-1705. b) A. Marimuthu, J. Zhang and S. Linic, Science, 2013, 339, 1590-1593. c) M. T. Pirnot, D. A. 70 Rankic, D. B. C. Martin and D. W. C. MacMillan, Science, 2013, 339, 1593-1596.

2. a) J. Rosenthal, T. D. Luckett, J. M. Hodgkiss, and D. G. Nocera, $J$. Am. Chem. Soc., 2006, 128, 6546-6547. b) L. A. Campbell, J. Mol. Cat. A: Chem. 1996, 1169. c) Z. Gross and S. Ini, J. Org. Chem., 1997, 3263, 5514-5521.

3. a) X.-T. Zhou, Q.-H. Tang, and H.-B. Ji, Tetrahedron Lett., 2009, 50, 6601-6605. b) R. Krishnan and S. Vancheesan, J. Mol. Catal. A: Chem., 2002, 185, 87-95.

4. Z. Xiantai and J. Hongbing, Chem. Eng. J., 2010, 156, 411-417.

805 . a) S. Cantekin, T. F. A. de Greef and A. R. A. Palmans, Chem. Soc. Rev., 2012, 41, 6125-6137. b) A. J. Wilson, M. Masuda, R. P. Sijbesma, and E. W. Meijer, Angew. Chem., Int. Ed. Engl., 2005, 44, 2275-2279.

6. R. van Hameren, P. Schön, A. M. van Buul, J. Hoogboom, S. V 85 Lazarenko, J. W. Gerritsen, H. Engelkamp, P. C. M. Christianen, H. 
A. Heus, J. C. Maan, T. Rasing, S. Speller, A. E. Rowan, J. A. A. W. Elemans, and R. J. M. Nolte, Science, 2006, 314, 1433-1436.

7. a) M. Hajimohammadi, N. Safari, H. Mofakham, and A. Shaabani, Tetrahedron Lett., 2010, 51, 4061-4065. b) K. Ishii, Coord. Chem. 5 Rev., 2012, 256, 1556-1568. c) R. Bonnet, Chem. Soc. Rev., 1995, 24, 19-33.

8. K. A. Prokop and D. P. Goldberg, J. Am. Chem. Soc., 2012, 134, 8014-8017.

9. N. J. In. Turro, Modern Molecular Photochemisry; Benjamin / Cummings: Menlo Park, CA, 1978, 579-611.

10 10. P. Thordarson, E. J. A. Bijsterveld, A. E. Rowan and R. J. M. Nolte, Nature, 2003, 424, 915-918. 